\section{Acknowledgement to Reviewers}

Oncol Res Treat 2021;44:719-720

DOI: $10.1159 / 000520911$

The editors extend their gratitude and appreciation to the following reviewers whose comments and criticism ensure the quality of the articles published in this journal.

Valentina Ambrosini, Bologna, Italy Leslie Andritsos, Albuquerque, NM, USA Silvia Antoine, Bochum, Germany Kayhan Azadmanesh, Tehran, Iran Ulli Simone Bankstahl, Frankfurt/M., Germany

Maggie Banys-Paluchowski, Lübeck, Germany

Jana Barinoff, Berlin, Germany Sebastian Bauer, Essen, Germany Meinrad Beer, Ulm, Germany Sebastian Belle, Mannheim, Germany Justus Benrath, Mannheim, Germany Lothar Bergmann, Frankfurt/M., Germany

David Blum, Zurich, Switzerland Stefan Boeck, Munich, Germany Jan Bornschein, Oxford, UK Walburgis Brenner, Mainz, Germany Paul Bröckelmann, Cologne, Germany Peter Brossart, Bonn, Germany Marco Cerrano, Turin, Italy Geothy Chakupurakal, Koblenz, Germany

Thomas Decker, Ravensburg, Germany

Barbara Deschler-Baier, Würzburg, Germany

Tony Dhillon, Guildford, UK

Nina Ditsch, Augsburg, Germany Karolina Edlund, Dortmund, Germany

Caroline Eich, Kleve, Germany

Michael Hans Robert Eichbaum, Wiesbaden, Germany

Dennis Eichenauer, Cologne, Germany Khaled Elsayad, Münster, Germany Thomas Ettrich, Ulm, Germany Falco Fend, Tübingen, Germany Nikolaj Frost, Berlin, Germany Cordula Gebel, Jena, Germany Franziska Geiser, Bonn, Germany Voica Ghilescu, Heidenheim, Germany Thorsten Oliver Götze, Frankfurt/M., Germany

Michael Golatta, Heidelberg, Germany

Heiko Golpon, Hannover, Germany Martin Gramatzki, Kiel, Germany Eva-Maria Grischke, Tübingen, Germany Viktor Grünwald, Essen, Germany Georg Martin Haag, Heidelberg, Germany
Stephan Hackenberg, Würzburg, Germany

Daniel Hartmann, Munich, Germany

Steffen Hauptmann, Mönchengladbach, Germany

Matthias Heck, Munich, Germany Marcus Hentrich, Munich, Germany Dominik Hering, Münster, Germany Marco Herling, Leipzig, Germany Tobias Herold, Munich, Germany Inken Hilgendorf, Jena, Germany Andreas Hochhaus, Jena, Germany Thomas Hundsberger, St. Gallen, Switzerland

Xingyuan Jiao, Guangzhou, China Sven Jonas, Berlin, Germany Martin Kaiser, London, UK

Bernd Kasper, Mannheim, Germany

Diego Kauffmann-Guerrero, Munich, Germany

Matthias Kaufmann, Erlangen, Germany

Ulrich Keller, Berlin, Germany

Elham Khatamzas, Munich, Germany

Rüdiger Klapdor, Hannover, Germany

Martin Kocher, Cologne, Germany

Attila Kollar, Bern, Switzerland

Martin Kortüm, Würzburg, Germany

Julia Kovacs, Munich, Germany

Anna-Lena Kraeft, Bochum, Germany

Matthias Kroiss, Munich, Germany

Utz Krug, Leverkusen, Germany

Harald Kühl, Munich, Germany

Walther Kuhn, Deggendorf, Germany

Volker Kunzmann, Würzburg, Germany

Elena Laakmann, Hamburg, Germany

Annette Lebeau, Hamburg, Germany

Andreas Leithner, Graz, Austria

Gerda Charlotte Leitner, Vienna, Austria

Florian Lordick, Leipzig, Germany

Diana Lüftner, Berlin, Germany

Celine Lugnier, Bochum, Germany

Manfred P. Lutz, Saarbrücken, Germany

Aline Maehringer-Kunz, Mainz, Germany Javier Martínez Trufero, Zaragoza, Spain

Aikaterini Mastoraki, Athens, Greece

Sibylle C. Mellinghoff, Cologne, Germany

Harri Merisaari, Turku, Finland

Maximilian Merz, Leipzig, Germany

Klaus H. Metzeler, Munich, Germany 
Moritz F. Meyer, Essen, Germany Volkmar Müller, Hamburg, Germany Gabriele Multhoff, Munich, Germany Markus Munder, Mainz, Germany Wakana Murakami, Tokorozawa, Japan Nina Rosa Neuendorff, Essen, Germany Guenter Niegisch, Düsseldorf, Germany Lucia Nogoova, Cologne, Germany Michael Oertel, Münster, Germany Oliver Ott, Erlangen, Germany Pia Paffenholz, Cologne, Germany Tjoung Won Park, Hannover, Germany Wolfgang Parsch, Nürnberg, Germany Roman Pfister, Cologne, Germany David Pfister, Cologne, Germany Daniel Pink, Bad Saarow, Germany Till Plönes, Essen, Germany Ilinca Popp, Freiburg i.Br., Germany Marc Raab, Heidelberg, Germany Christina Ramsenthaler, Freiburg i.Br., Germany

Peter Reichardt, Berlin, Germany

Daniel Reim, Munich, Germany Toralf Reimer, Rostock, Germany Jan Rémi, Munich, Germany Daniel Rolf, Münster, Germany David Santamaria, Bordeaux, France Bastian Schilling, Würzburg, Germany Mona Wanda Schmidt, Mainz, Germany Lars Henning Schmidt, Ingolstadt, Germany

Patrick Schöffski, Leuven, Belgium Christoph Scholz, Munich, Germany Sergiu Scobioala, Münster, Germany Martin Sebastian, Frankfurt/M., Germany
Christoph Seidel, Hamburg, Germany Laura Sellmer, Munich, Germany Tanja Sprave, Freiburg i.Br., Germany Alexander Stein, Hamburg, Germany Juergen Michael Steinacker, Ulm, Germany

Kathrin Britta Stewen, Mainz, Germany Sebastian Stintzing, Berlin, Germany Gertraud Stocker, Leipizig, Germany Jana Kaethe Striefler, Berlin, Germany Herwig Strik, Bamberg, Germany Sebastian Theurich, Munich, Germany Ali Vahidirad, Mashhad, Iran Birgitt van Oorschot, Würzburg, Germany

Yogesh Vashist, Burghausen, Germany Marie von Lilienfeld-Toal, Jena, Germany Martin Wagner, Ulm, Germany Julia Walter, Munich, Germany Rudolf Weide, Koblenz, Germany Arndt Weinmann, Mainz, Germany Ellen Weisberg, Boston, MA, USA

Anja Welt, Essen, Germany

Christoph Benedikt Westphalen, Munich, Germany

Marcel Wiesweg, Essen, Germany

Mathias Witzens-Harig, Heidelberg, Germany

Bernhard Wörmann, Berlin, Germany

Ursula Wolf, Bern, Switzerland

Rachel Würstlein, Munich, Germany

Perran Fulden Yumuk, Istanbul, Turkey

Thomas Zander, Cologne, Germany Tianzuo Zhan, Mannheim, Germany Sarah Zierold, Munich, Germany 